Pediat. Res. 6: 659-663 (1972)

Amino acids amniotic fluid

\title{
Amino Acids in Amniotic Fluid: Changes in Concentrations During the First Half of Pregnancy
}

\author{
C. Ronald Scott ${ }^{[17]}$, Gecilia Ghiang Teng, Ronald N. Sagerson, and Thomas Nelson \\ Department of Pediatrics, School of Medicine, University of Washington, Seattle, Washington, USA
}

\begin{abstract}
Extract
The free amino acids in amniotic fluid were quantitated by ion-exchange chromatography in human fetuses of 48- to 140-day gestation (mean, 91 days). Gestational age was estimated by crown-rump measurement. The amino acid pattern was more similar to that found in normal plasma than to that usually observed in urine. Fourteen of 26 amino acids (lysine, leucine, phenylalanine, isoleucine, valine, ornithine, alanine, tyrosine, methionine, arginine, serine, histidine, proline, and taurine) showed a statistically significant change in concentration $(P<0.005)$ which correlated inversely with fetal age (Fig. 1). Alanine had the highest average concentration during early gestation, $0.437 \mathrm{~mm}$. The concentration of lysine showed the highest degree of correlation with fetal age $(P \ll 0.001)$, with a correlation coefficient of 0.89 . The most highly correlated single-regression curve was for lysine; where [lys] = 0.796-0.0052 (fetal age). None of the amino acid concentrations increased during the period of observation.
\end{abstract}

\section{Speculation}

It has been suggested that the concentrations of constituents in amniotic fluid may serve as an indicator of fetal genotype and be of value in antenatal diagnosis. Because of the magnitude of the changes in amino acid concentration in amniotic fluid that occur during early gestation, accurate methods of fetal dating will be necessary if amino acid concentration is to be used for antenatal diagnostic purposes. The results of this study would suggest that regression analysis of amino acid concentration in amniotic fluid may prove to be an independent and reliable estimator of fetal age.

\section{Introduction}

The free amino acids in amniotic fluid may be of diagnostic value for fetuses at risk for inborn errors of amino acid metabolism. Before amino acid concentrations can be used as an indicator of fetal genotype, it is mandatory that normal amino acid values be obtained and correlated with fetal gestational age. This study was undertaken to determine the concentrations of free amino acids in amniotic fluid and to determine which amino acids would offer the best correlation with fetal age.

\section{Methods}

Amniotic fluid was obtained during therapeutic abortions performed by hysterotomy. In each case, the pregnant woman was hospitalized overnight and fasted 
8-12 hr before the hysterotomy. None of the pregnancies was terminated for suspected fetal disorders. Immediately after the hysterotomy, amniotic fluid free of maternal tissue contamination was obtained by needle aspiration of the amniotic sac. Twenty-four samples were used for the study.

Gestational age of the fetus was estimated by crownrump measurement. Correlation of crown-rump length to gestational age was based on previously studied fetal material obtained from therapeutic abortions [8, 10]. Gestational age was calculated from the time of fertilization.

Amino acids were quantitated by ion-exchange chromatography according to the method of Spackman et al. [9]. An amino acid analyzer [12] was used for all analyses. One $\mathrm{ml}$ fresh amniotic fluid was treated with $1 \mathrm{ml} 6 \%$ sulfosalicylic acid buffered to $\mathrm{pH} 2$ and was then centrifuged; the protein-free supernatant was used for amino acid analysis. When protein could not be precipitated immediately, the amniotic fluid was frozen at $-20^{\circ}$ and subsequently analyzed within 48 $\mathrm{hr}$. The equivalent of $0.25 \mathrm{mI}$ aminotic fluid was routinely applied to the ion-exchange column.

A biomedical linear regression program [13] was used for calculation of the regression curves, correlation coefficients, mean values, and standard deviations of the amino acid concentrations [1].

\section{Results}

A significant quantity of free amino acids was found in each amniotic fluid sample. The amino acids were clearly separated and more similar to the pattern seen in normal plasma than to that usually observed in urine. There were few, if any, unidentifiable peaks which contributed significantly to the ninhydrin posi-

Table I. The amino acids in amniotic fluid which did not significantly change in concentration as a function of fetal age $(P>0.05)^{1}$

\begin{tabular}{ll}
\hline \multicolumn{1}{c}{ Amino acid } & Concentration, $\mathrm{ms}^{2}$ \\
\hline Aspartic acid & $0.007 \pm 0.011$ \\
Threonine & $0.196 \pm 0.047$ \\
Glutamine & $0.296 \pm 0.125$ \\
Glutamic acid & $0.165 \pm 0.084$ \\
Gitrulline & $0.012 \pm 0.022$ \\
Glycine & $0.172 \pm 0.050$ \\
1/2 Cystine & $0.051 \pm 0.026$ \\
Cystathionine & $0.002 \pm 0.003$
\end{tabular}

1 Range of gestational age, 48-140 days; average, 91 days.

${ }^{2}$ Mean $\pm \mathrm{SD}$. tive material. Tryptophane is not accurately quantitated by ion-exchange chromatography and was not independently assayed. Glutamine and asparagine eluted as a single peak under the conditions of analysis; however, they can be separated by thin-layer chromatography (TLC). The TLC revealed little asparagine; therefore, the peak obtained by column chromatography was assumed to be primarily glutamine. Twenty-six amino acids could be consistently identified in the fluid. Twenty-two of these were quantitated in each specimen. Alpha-amino- $n$-butyric acid, hydroxyproline, phosphoethanolamine, and ethanolamine were usually present in low concentrations and were not quantitated. Occasionally, 1-methyl-histidine or homocarnosine were identified.

It was apparent that several of the amino acids were present in greater concentration early in fetal life whereas others showed no significant change during the first half of pregnancy. Table I lists the concentrations of the eight amino acids for which there was no statistical relation between fetal age and amino acid concentration. For the other 14 amino acids, a statistical relation between fetal age and concentration in aminiotic fluid could be demonstrated. The concentrations of lysine, leucine, valine, ornithine, isoleucine, phenylalanine, alanine, methionine, arginine, tyrosine and serine were correlated with gestational age at the 0.001 level of statistical significance, while histidine, proline and taurine rendered significance at the 0.005 level of significance (Table II). This is depicted in Figure 1.

The change in concentration between 48 and 140 days was not great for phenylalanine, tyrosine, methionine, serine, ornithine and arginine, but the precision of measurement permitted a significant correlation with fetal age. Changes were more striking for the branched-chain amino acids, leucine, isoleucine, and valine. They showed significant decline during the early part of pregnancy with valine having the greatest rate of change. Another feature was the positive correlation of the concentration of these branched-chain amino acids within the same amniotic fluid sample. Thus, if the concentration of leucine in aminiotic fluid was known, the partial correlation coefficients to valine and isoleucine were 0.98 and 0.99 respectively. The fact that common or similar enzymes are involved in the degradation of these three amino acids may account for this high degree of correlation.

The concentration of lysine showed the highest degree of correlation with fetal age $(P \ll 0.001)$ and had a correlation coefficient of 0.89 . Alanine had the high- 
Table II. The amino acids which showed a statistically significant correlation between their concentrations in amniotic fluid and the gestational age of the fetus ${ }^{2}$

\begin{tabular}{|c|c|c|c|}
\hline Amino acid & $\underset{\mathrm{m} M L}{\text { Mean }}$ concentration, & Regression equation $\pm 95 \%$ confidence limit & F Value ${ }^{2}$ \\
\hline \multicolumn{4}{|c|}{ Significant at $P<0.001$ level: } \\
\hline Lysine & 0.313 & $0.796-0.0052 x \pm 0.151$ & $76.6^{3}$ \\
\hline Leucine & 0.129 & $0.331-0.0022 \chi \pm 0.080$ & 54.1 \\
\hline Phenylalanine & 0.077 & $0.160-0.00092 x \pm 0.034$ & 49.8 \\
\hline Isoleucine & 0.057 & $0.145-0.00096 x \pm 0.036$ & 49.6 \\
\hline Valine & 0.229 & $0.518-0.0032 x \pm 0.124$ & 45.2 \\
\hline Ornithine & 0.048 & $0.125-0.00082 \chi \pm 0.032$ & $39.3^{4}$ \\
\hline Alanine & 0.437 & $0.837-0.0044 \chi \pm 0.188$ & 37.7 \\
\hline Tyrosine & 0.071 & $0.143-0.00079 x \pm 0.034$ & 36.4 \\
\hline Methionine & 0.025 & $0.048-0.00025 x \pm 0.011$ & 32.8 \\
\hline Arginine & 0.054 & $0.184-0.0014 \chi \pm 0.065$ & $24.2^{4}$ \\
\hline Serine & 0.038 & $0.098-0.00066 x \pm 0.042$ & 17.2 \\
\hline \multicolumn{4}{|c|}{ Significant at $0.001<P<0.005$ level: } \\
\hline Histidine & 0.097 & $0.164-0.00072 x \pm 0.053$ & $11.7^{3}$ \\
\hline Proline & 0.219 & $0.387-0.0018 x \pm 0.143$ & 11.4 \\
\hline Taurine & 0.143 & $0.254-0.0012 x \pm 0.098$ & 10.6 \\
\hline
\end{tabular}

1 Range of gestational ages, 48-140 days; average, 91 days.

2 df: $1,22$.

323 determinations, df: 1,21 .

421 determinations, df: 1,19 .

est average concentration during early gestation, 0.436 mM.

\section{Discussion}

Levy and Montag [4] were the first to report the use of ion-exchange chromatography for the quantitation of free amino acids in amniotic fluid, and they determined the normal concentrations of free amino acids at birth in comparison with the values in maternal and neonate plasma. Several subsequent reports have documented the concentrations of amino acids in amniotic fluid obtained primarily by transabdominal amniocentesis between the eighth gestational week and birth $[2,5-7,11]$. In each report the date of the last menses was used as the best indicator of fetal age. Several of the papers have recognized the general association between amino acid concentration and gestational age and the values were reported by trimesters or by various weekly intervals. None have recognized the very strong correlation between fetal age and the concentrations of specific amino acids in amniotic fluid. This may have occurred because of the relative inaccuracy of using menstrual history for fetal dating.

The results of the present study convincingly demonstrate that early in pregnancy some amino acids are present in amniotic fluid in relatively high concentrations which decrease during the first half of pregnancy.
Those amino acids which decreased in concentration did so as a function of fetal age.

It has been suggested that the concentration of constituents in amniotic fluid may serve as an indicator of fetal genotype and thus be of value in antenatal diagnosis. The present results offer normal values for the concentrations of amino acids during the first half of pregnancy when therapeutic intervention would be most logical. Because of the magnitude of the changes in amino acid concentrations during the first half of pregnancy, accurate data based upon precise fetal age are important. Dating of amniocentesis specimens by menstrual history may not be precise enough to predict a specific amino acid concentration range and caution must be exercised in any clinical decisions that are based upon the concentrations of amino acids in amniotic fluid for antenatal diagnostic purposes.

The first attempt to use amino acids in amniotic fluid as a diagnostic indicator of fetal genotype was by Frimpter and coworkers [3] who monitored the pregnancy of a woman who had previously borne a child with cystathioninuria. Increased concentration of cystathionine was not detected in the amniotic fluid, and at delivery the child was found to be normal for cystathionine metabolism. O'Neill and associates [5] documented that an infant with maple syrup urine disease did not have increased concentrations of leucine, isoleucine or valine in the amniotic fluid at term. Con- 

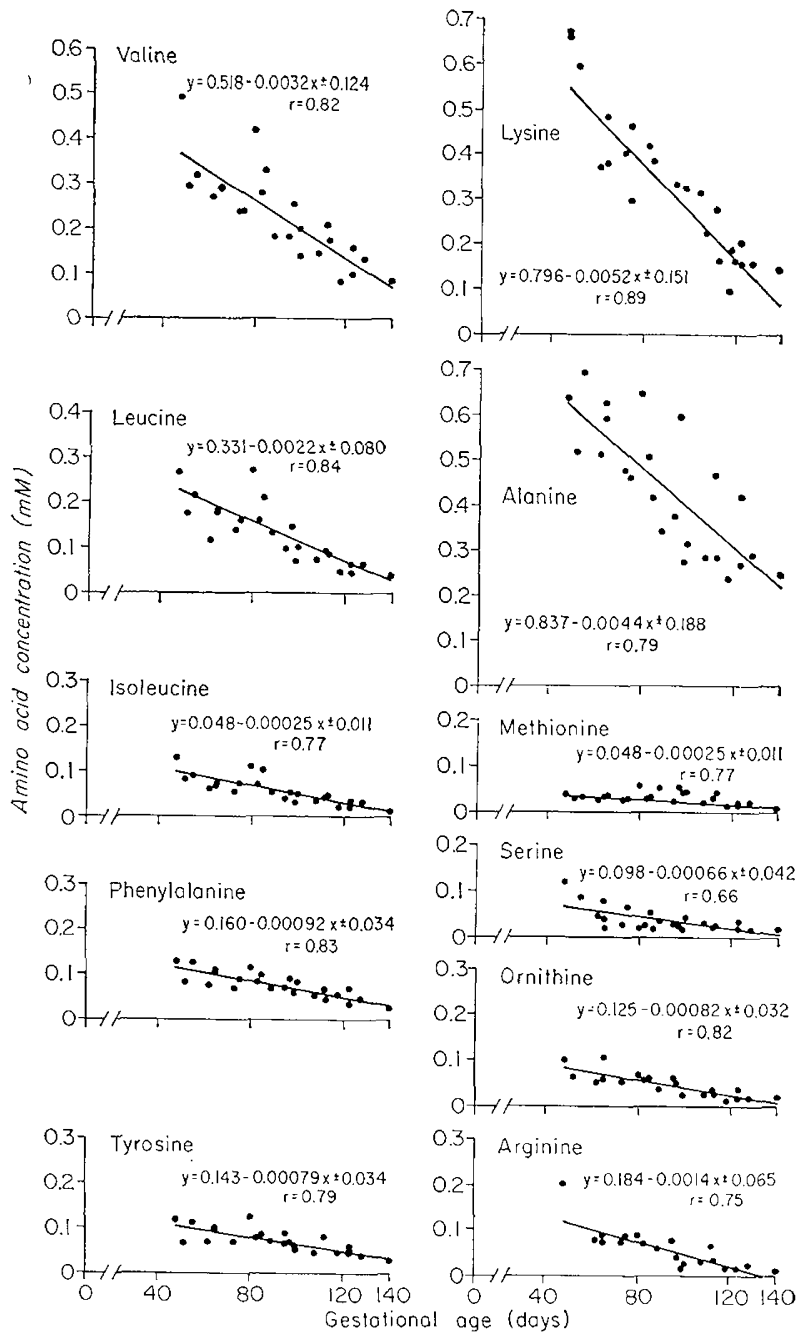

Fig. 1. Regression curves of the nine amino acids which showed a statistically significant correlation $(P<0.001)$ with gestational age. The equation indicates the best straight line obtained by the maximum likehood method ( $\pm 95 \%$ confidence limit) which fits the coordinates of fetal age (gestational age in days from fertilization) and amino acid concentration (ma). The expected amino acid concentration (y) can be calculated if the fetal age $(x)$ is known, or vice-versa. The correlation coefficient is given by $r$.

centrations of branched-chain amino acids in serum did not become elevated until three days of age. Whether amino acid concentrations in amniotic fluid can be a reliable indicator of infants homozygous for inborn errors of amino acid metabolism will need to be carefully evaluated. It is likely that heterozygous mothers may adequately regulate the concentrations of amino acids in plasma of infants homozygous for inborn errors of amino acid metabolism and that little alteration in the free amino acids of the amniotic fluid may be apparent. This may not be true, however, for infants with renal tubular transport defects.

An important question remaining is whether the concentrations of amino acids in amniotic fluid obtained by transabdominal amniocentesis can serve as an independent estimator of fetal age during the first half of pregnancy; if so, it would be an estimater of greater accuracy than menstrual history. This is being approached by a separate prospective study using the data from this study to generate the best regression equation to predict fetal age.

\section{Summary}

The concentrations of 22 amino acids in amniotic fluid were quantitated from 24 early pregnancies between 48 and 140 days of gestation. A strong statistical correlation was found between 14 amino acids and fetal age. This relation was inversely proportional to fetal age. Lysine showed the strongest correlation $(P \ll$ 0.001 ) and had a correlation coefficient of 0.89 . None of the amino acids increased in concentration during the time period of the study. There was a striking partial correlation between the branched-chain amino acids, leucine, isoleucine and valine, presumably because of the similar enzymes required for their metabolism.

The results of this study would suggest that early in pregnancy the concentrations of amino acids in amniotic fluid may prove to be an independent and reliable indicator of fetal age.

\section{References and Notes}

1. Drxon, W. J.: BMD Biomedical Computer Programs. (University of California Press, Berkeley, 1967).

2. Emery, A. E. H., Burt, D., ANd Nelson, M. M.: Antenatal diagnosis and amino acid composition of amniotic fluid. Lancet, $i$ : 1307 (1970).

3. Frimpter, G. W., Greenberg, A. J., Hrlgarten, M., and Fuchs, F.: Cystathioninuria: management. Am. J. Dis. Child., 113: 115 (1967)

4. Levy, H. L., AND MonTAG, P. P.: Free amino acids in human amniotic fluid. A quantitative study by ion-exchange chromatography. Pediat. Res., 3: 113 (1969).

5. O'Neill, R. T., Marrow, G., III, Hammel, D., Auerbach, V. H., AND BARNess, L. A.: Diagnostic significance of amniotic fluid amino acids. Obstet. Gynecol., 37: 550 (1971).

6. ReID, D. W. J., CaMpbell, D. J., and YaKYMYSHYN, L.Y.: Quantitative amino acids in amniotic fluid and maternal plasma in early and late pregnancy. Am. J. Obstet. Gynecol., III: 251 (1971).

7. Saifer, A., A'Zary, E., Valenti, C., And Schneck, L.: Quantita- 
tive cation-exchange chromatographic analysis of free amino acids in human amniotic fluid collected during early preg. nancy. Clin. Chem., 16: 891 (1970).

8. Shepard, T. H.: Growth and development of the human embryo and fetus. In: L. I. Gardner: Endocrine and Genetic Diseases of Childhood. (W. B. Saunders Co., London, 1969).

9. Spackman, D. H., Stern, W. H., ANd Moore, S.: Automatic recording apparatus for use in chromatography of amino acids. Anal. Chem., 30: 1190 (1958).

10. Tanimura, T., Nelson, T., Hollingsworth, R. R., and SHEPARD, T. H.: Weight standards for organs from early human fetuses. The Anatomical Record, 171: 227 (1971).

11. Thomas, G. H., Parmley, T. H., Stevenson, R. E., AND Howell, R. R.: Developmental changes in amino acid concentrations in human amniotic fluid: abnormal findings in maternal phenylketonuria. Am. J. Obstet. Gynecol., III: 38 (1971).
12. Spinco model 120C, Beckman Spinco, Palo Alto, Calif.

13. Adapted to the IBM 6400 computer, IBM Corporation, Data Processing Division, White Plains, N.Y.

14. Informed consent was obtained in accordance with the provisions set forth in the Declaration of Helsinki.

15. The authors are grateful to Dr. Thomas Shepard for his aid in the collection and dating of fetal specimens and to $\mathrm{Dr}$. Paula Diehr for her assistance in using the IBM $6400 \mathrm{com}$ puter.

16. This work was supported by Children's Bureau Grant no. DHEW-CB-913, National Institute of Child Health and $\mathrm{Hu}$ man Development Grant no. HD 04665, and National Foundation Grant no. CRBS-258.

17. Requests for reprints should be addressed to: C. Ronald ScotT, M.D., Department of Pediatrics, School of Medicine, University of Washington, Seattle, Washington 98195 (USA)

18. Accepted for publication February 15, 1972. 